\title{
Philosophiques
}

\section{Max Kistler, Causalité et lois de la nature , Paris, Vrin, 1999,} 311 p.

\section{Benoit Hardy-Vallée}

Volume 28, numéro 2, automne 2001

URI : https://id.erudit.org/iderudit/005668ar

DOI : https://doi.org/10.7202/005668ar

Aller au sommaire du numéro

Éditeur(s)

Société de philosophie du Québec

ISSN

0316-2923 (imprimé)

1492-1391 (numérique)

Découvrir la revue

Citer ce compte rendu

Hardy-Vallée, B. (2001). Compte rendu de [Max Kistler, Causalité et lois de la nature , Paris, Vrin, 1999, 311 p.] Philosophiques, 28(2), 447-450.

https://doi.org/10.7202/005668ar d'utilisation que vous pouvez consulter en ligne.

https://apropos.erudit.org/fr/usagers/politique-dutilisation/ 


\section{Max Kistler, Causalité et lois de la nature, Paris, Vrin, 1999, 311 p.}

Causalité et lois de la nature, de Max Kistler est un ouvrage destiné à devenir un nouveau classique. Il trouvera place dans la bibliothèque du philosophe des sciences aux côtés des Structure des révolutions scientifiques (Kuhn), Logique de la découverte scientifique (Popper) et autre Structure of science (Nagel). Il sera sans doute un nouveau classique parce qu'il est, en un autre sens, un nouveau classique : l'auteur, par-delà le nominalisme propre à Hume et à la modernité, retrouve une pensée scientifique authentiquement réaliste. L'auteur affranchit la causalité des connotations subjectives habituelles, ce qui devrait susciter moultes réflexions philosophiques et scientifiques.

Le livre est clairement un ouvrage pour philosophes expérimentés : la profondeur et la complexité des questions rebuteraient le néophyte mais pourraient par là même stimuler le désir d'explorer les problématiques. Les sujets abordés sont d'une importance capitale et l'auteur leur fait honneur par une présentation claire et détaillée des enjeux, arguments et contre-arguments. Il offre de plus un panorama exhaustif de la philosophie des sciences actuelle.

Le concept de causalité, dans l'optique de l'auteur, doit être reconstruit pour être à la fois objectif et adapté à la science contemporaine. Il faut comprendre : l'ensemble de la science et non pas uniquement la physique des particules.

Les interprétations nominalistes habituelles du concept de causalité, qu'elles soient instrumentalistes ou psychologistes, s'entendent généralement pour en faire un concept trop intuitif et utilitaire pour être objectif et adapté à la science. On n'a qu'à penser à la parole de Russell, qui comparait la causalité à la monarchie anglaise : elle n'a survécu que parce qu'on croit, à tort, qu'elle ne fait pas de dégâts. Quant à Nagel, il préfère rejeter ce concept en raison de sa polysémie intrinsèque.

Pourquoi s'obstiner à retrouver ce concept ? Contrairement à Aristote, Kistler n'entend pas fournir un concept de causalité qui s'applique à toutes les explications de phénomènes naturels. Ce concept sera utile seulement s'il est à même de fournir une distinction pertinente entre les explications causales et non causales. Cette recherche ne sera pas le fruit d'une analyse a priori du terme "causalité ", ni d'une observation des pratiques linguistiques où apparaît ce terme. Plutôt, en philosophe post-quinien, Kistler forge une conception a posteriori, qui ne prétend ni à l'analyticité ni à l'irréfutabilité. Son concept sera soumis au double impératif naturaliste, c'est-à-dire de convenir à une panoplie de phénomènes de types différents tout en conservant un sens véritablement naturaliste : réductionniste quant aux objets mais non-éliminativiste quant aux propriétés. L'auteur épouse une position qui ne nie pas le monisme ontologique tout en reconnaissant que des propriétés non-microphysiques puissent être causalement efficaces.

Kistler présente tout d'abord (chapitre 1) sa conception réaliste de la causalité et son lien avec les lois de la nature. Point de départ obligé, l'analyse de Kistler commence par Hume. Ce dernier est en effet à l'origine de la conception moderne de la causalité : il n'y a pas de «colle métaphysique » qui relie de manière nécessaire deux événements par une relation de cause à effet mais plutôt une habitude cognitive, et donc ce concept n'a pas de fondement objectif. Cette habitude consisterait, pour certains des événements successifs et contigus, à leur attribuer l'idée qu'il y a une connexion nécessaire entre eux lorsque cette succession a déjà eu lieu. Le jugement causal dépendrait donc de l'observateur et non de l'observé (p. 25). La philosophie 
analytique des sciences, par le modèle nomologico-déductif (essentiellement de nature logique) de l'explication, s'inscrivait dans la même ligne de pensée humienne, si bien qu'on enseigne aujourd'hui l'épistémologie comme si cet anti-réalisme allait de soi. Quine ne disait-il pas que la condition humienne est la condition humaine ?

Kistler n'est pas le premier à proposer une alternative à cet empirisme subjectif : la théorie singulariste (Anscombe, Ducasse) et la théorie anthropocentriste (Gasking) visaient à répondre au défi sceptique. Dans le premier cas, on conçoit qu'il y a causalité en autant qu'un événement individuel exerce une influence efficace sur un autre, sans pour autant supposer qu'il y ait de connexion nécessaire ni de récurrence. Mais considérer que la causalité n'est pas la nécessité est insuffisant pour expliquer de façon positive ce qu'est une relation de causalité et pour rendre compte, au-delà de la succession et de la contiguïté, de l'asymétrie entre cause et effet. Dans l'autre cas, la causalité est en fait une projection de nos comportements dans l'interprétation des comportements. Nous attribuerions aux phénomènes naturels des propriétés causales mais uniquement de façon dérivée : tout énoncé causal peut être reformulé en termes d'action humaine. Le problème est qu'on a alors expliqué le général par le particulier, ce qui ne correspond pas à ce qu'on entend généralement par « explication ». L'auteur s'en tiendra plutôt à la théorie dite " de transfert " :

(S) Deux événements $c$ et $e$ sont liés comme cause et effet si et seulement s'il existe au moins une grandeur physique $\mathrm{P}$, soumise à une loi de conservation, exemplifiée dans $c$ et $e$, et dont une quantité déterminée est transférée entre $c$ et $e$.

La théorie réaliste de la causalité s'articulera donc en trois parties : la première constitue la théorie présentée ici, dans laquelle la causalité est une relation de transfert d'énergie. Deuxièmement, il y a relation causale lorsque sont instanciées des propriétés d'événements et finalement lorsque l'instanciation d'une première propriété entraîne la seconde en vertu d'une loi de la nature.

À la différence des analyses conceptuelles traditionnelles, celle-ci ne prétend pas être a priori et est donc réfutable. Ainsi, l'antériorité de la cause vis-à-vis de l'effet ne provient pas de la définition de «causalité »: il s'agit "plutôt d'une circonstance contingente qui peut, par ailleurs, se révéler fausse. » (p.49)

La relation causale peut donc être réduite non pas à la structure logique de l'explication scientifique mais, a posteriori, aux propriétés physiques des événements puisqu'on en parle en termes de transfert d'énergie. La causalité est délivrée de l'expérience : on n'a pas à l'expliquer uniquement en termes observationnels et ainsi l'explication peut faire intervenir des termes théoriques. La cause et l'effet sont tels selon la direction du transfert d'énergie et ils ne sont plus tributaires de l'observateur puisqu'on en parle en termes de propriétés d'événements.

Les lois de la nature (chapitre 2) seront aussi comprises d'un point de vue réaliste plutôt qu'en terme de modèles ou de normes. Les généralisations universelles renvoient non pas, contra Frege, à des concepts, mais à des instanciations de propriétés dans le cas des lois de la nature ou à des objets dans le cas de généralisations accidentelles. Le problème de la similarité syntaxique entre ces deux types de généralisations (nomologiques et accidentelles) est donc résolu sémantiquement par la différence d'interprétation.

Afin de présenter une conception de la causalité qui satisfasse au double impératif naturaliste mentionné plus haut, l'auteur doit rejeter la dichotomie entre les lois dites strictes et les lois ceteris paribus (chapitre 3) : le modèle d'explication causale 
s'applique à tout type de relation causale. Il utilise la théorie des perturbations à cette fin : cette métathéorie (p.177) dicte la classification quantifiée des facteurs qui influencent un événement pour isoler les perturbations. De la sorte, les exceptions à une loi sont dues à des lois différentes de la première : il y a exception à la première lorsque les autres concourent à interférer avec elle.

Les quatrième et cinquième chapitres introduisent le concept de responsabilité causale, crucial pour l'argumentation de l'auteur, de même que la structure logique de ce concept. Dans une relation causale telle qu'il l'entend, l'instanciation d'une première propriété est causalement responsable de l'instanciation d'une seconde. Il y a donc deux niveaux conceptuels dans une explication causale : la relation elle-même entre des propriétés d'événements par le biais d'un transfert d'énergie, et la relation de responsabilité causale entre des faits d'instanciations de propriétés qui rendent vraie l'explication causale. La distinction entre un fait et un événement est essentielle : l'événement a une structure uniquement spatio-temporelle, alors que le fait est la prédication vraie d'un événement. Pour réduire une relation de causalité aux propriétés physiques d'événements, il faut pouvoir réduire un énoncé causal factuel à un énoncé causal événementiel. C'est la nature du transfert d'énergie qui déterminera le contenu des énoncés causaux factuels : lorsqu'un glaçon fond, l'énergie va de l'eau ambiante vers l'eau du glaçon et non l'inverse. Cet événement rendra vrai l'énoncé affirmant que le fait que l'eau émet de la chaleur est la cause du fait que le cube de glace fond.

La responsabilité causale est un concept plus riche que la causalité elle-même en ce qu'il fait intervenir l'idée que, entre un fait antécédent et un fait conséquent, il y a une relation de responsabilité lorsqu'une loi de la nature s'applique à la situation.

On peut exprimer formellement la structure des énoncés causaux factuels par :

(1) (F) (G) CF (Fc, Ge)

et celle des énoncés causaux événementiels par :

(2) $(\mathrm{F})(\mathrm{G}) \mathrm{CE}(c, e)^{\wedge} \mathrm{Fc}^{\wedge} \mathrm{Ge}^{\wedge} \mathrm{L}(\mathrm{F}, \mathrm{G})(\mathrm{p} .19)$

(2) exprime un rapport d'efficacité causale, telle que spécifié par (S), entre un événement $c$ qui a la propriété $\mathrm{F}$ et un événement $e$ qui a la propriété $\mathrm{G}$ selon une loi de la nature d'après laquelle une instanciation de G-ité découle d'une instanciation de F-ité. Ces propriétés, pour avoir une efficacité causale, doivent être de type intrinsèques, c'est-à-dire non-relationnelles : des propriétés qui s'appliquent à l'espacetemps occupé par l'événement (chapitre 6).

Il n'y a donc plus d'adéquation simple entre l'explication nomologicodéductive et l'explication causale : la structure logique de la seconde et l'asymétrie entre la cause et l'effet ne sont pas reflétées dans la première. De plus, nombre d'explications ne sont pas causales : l'explication mathématique en termes d'axiomes, l'explication de la température d'un gaz par sa pression, l'explication de la température par la réduction à la moyenne de l'énergie moléculaire des composantes, etc.

Quant au septième et dernier chapitre, il se révèle des plus riches : l'auteur y expose, avec la même rigueur qui parcourt le livre, la fécondité de son concept de responsabilité causale. 
Kistler réussit à atteindre les objectifs qu'il s'était fixés au début du livre. L'un d'eux, cependant, reste encore en suspens : bien que l'auteur ait réussi à forger le concept de causalité (et de responsabilité causale) afin de l'appliquer à l'ensemble des sciences, sa réflexion ne semble pas s'émanciper du domaine de la physique fondamentale. Cela ne signifie pas qu'on ne peut pas utiliser sa théorie en psychologie ou en sciences sociales, mais qu'on ne sait pas encore comment. Si la causalité se réduit au transfert d'énergie, il serait intéressant de voir comment les transferts d'énergie interviennent dans ces sciences spéciales. 